\title{
Biodiversità di lieviti isolati da siero-innesto di produzioni casearie italiane
}

\author{
Yeast biodiversity in natural whey cultures for italian cheese \\ production
}

Istituto di Scienze delle Produzioni Alimentari, Consiglio Nazionale Ricerche, Milano, Italia

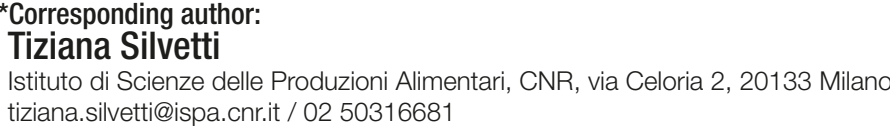

Ricevuto il 29 luglio 2019

Accettato il 24 settembre 2019

DOI: DOI: 10.36138/STLC.01.2019.03

\section{Riassunto}

\section{Obiettivi}

Studiare la biodiversità e il potenziale metabolico di lieviti isolati da siero-innesto naturale di Trentingrana DOP e Provolone Valpadana DOP.

\section{Materiali e metodi}

Da 34 siero-innesti (21 da Trentingrana e 13 da Provolone) sono stati isolati 54 ceppi di lieviti successivamente tipizzati (RAPD-PCR), identificati (sequenziamento ITS e D1/D2) e caratterizzati dal punto di vista tecnologico (attività lipolitica, proteolitica e produzione di biofilm).

\section{Risultati}

II contenuto di lieviti dei sieri-innesto era compreso tra 0.95 e 5.27 $\log _{10}$ UFC/mL. Gli isolati appartene- vano a 9 generi e 15 specie diverse. II $90 \%$ dei ceppi è risultato lipolitico, mentre il 30\% ha mostrato attività proteolitica. II 73\% dei ceppi era in grado di formare biofilm.

\section{Conclusioni}

II siero-innesto di produzioni casearie italiane DOP è caratterizzato da una ricca biodiversità di lieviti, che possiedono un potenziale metabolico in grado di influenzare fortemente la qualità e la sicurezza dei prodotti caseari.

\section{Parole chiave:}

Biodiversità

- Lieviti

Siero-innesto

Trentingrana DOP

Provolone DOP

\section{Abstract}

Objectives

Yeasts isolated from natural whey starters for Trentingrana PDO and Provolone Valpadana PDO have been identified, typed and technologically characterized to highlight their biodiversity and metabolic potential.

\section{Methods}

Fifty-four yeasts from 34 whey starters (21 from Trentingrana and 13 from Provolone) were isolated, subsequently typed (RAPD-PCR), identified (ITS and D1/D2 sequencing) and technologically characterized (lipolytic and proteolytic activity and biofilm production).

\section{Results}

The yeast content in whey starters varied between 0.95 and $5.27 \log _{10}$ $\mathrm{CFU} / \mathrm{mL}$. The isolates belonged to 9 genera and 15 species. Almost al strains were lipolytic, while 30\% showed proteolytic activity. A marked lipoand proteolytic potential by Aureobasidium pullulans emerged. Most strains (73\%) were able to form biofilm.

\section{Conclusion}

The whey starter of Italian PDO cheeses is characterized by a rich yeast biodiversity, possessing a metabolic potential that can strongly influence the quality and safety of dairy products.

\section{Keywords: \\ Biodiversity \\ - Yeast \\ - Whey starter \\ - Trentingrana PDO \\ Provolone PDO}




\section{INTRODUZIONE}

I siero-innesti sono caratterizzati da una relativa biodiversità nel numero di specie e da una notevole diversità in biotipi all'interno delle colture. La prevalenza di poche specie dominanti suggerisce che esse contribuiscano in modo significativo alle caratteristiche sensoriali e alla peculiarità dei formaggi DOP e tradizionali. La caratterizzazione dei siero-innesti è, dunque, essenziale per comprendere le dinamiche che avvengono all'interno della comunità microbica $\mathrm{d} i$ questo prodotto al fine di preservarne la ricchezza microbica e l'attività metabolica che determinano la qualità e sicurezza dei prodotti caseari (1).

In particolare, i lieviti possono rappresentare una parte importante della comunità microbica presente nell'ambiente del caseificio, nel latte crudo e anche nel siero-innesto naturale. Kluyveromyces marxianus, ad esempio, è specie molto frequente nel siero-innesto naturale utilizzato per la produzione di Parmigiano Reggiano (2).

I lieviti sono in grado di colonizzare la superficie dei formaggi a inizio stagionatura, formando unitamente ad altri microrganismi un biofilm complesso, e così partecipare attivamente al processo di caseificazione. Concorrono, dunque, in modo significativo alla definizione del sapore e all'aspetto tipico dei formaggi. Alcuni lieviti sono anche in grado di crescere all'interno del formaggio, producendo etanolo e anidride carbonica durante le prime fasi di maturazione. Tuttavia, i lieviti possono causare il deterioramento del formaggio, dando origine a difetti che variano da off-flavours a formazione di occhiature indesiderate e colorazioni anomale della cagliata, nel corso della stagionatura e della conservazione (3).

L'influenza dei lieviti presenti nel siero-innesti sul processo di stagionatura del formaggio non è ad oggi molto chiara. È probabile che, se presenti in concentrazione moderata, essi siano positivi per lo sviluppo dell'aroma attraverso la loro attività proteolitica e alla produzione di composti organici volatili. Tuttavia, la presenza di lieviti in numero elevato (superiori a $10^{5} \mathrm{UFC} / \mathrm{ml}$ ) può essere un segnale per i casari del rischio di gonfiore precoce, dovuto a fermentazione alcolica indesiderata (4).

In generale, è importante identificare i lieviti associati ai diversi alimenti e stabilire relazioni tra le caratteristiche degli alimenti e il contenuto e la diversità della popolazione di lieviti. Nonostante il loro ruolo chiave in caseificio, le informazioni relative alla biodiversità e all'attività di lieviti naturalmente presenti nei prodotti lattiero-caseari sono ancora scarse.

In questo studio è stata, quindi, condotta un'indagine per caratterizzare i lieviti presenti nel siero-innesto naturale di produzioni casearie italiane (Trentingrana DOP e Provolone Valpadana DOP). I lieviti oggetto di studio sono stati identificati, tipizzati e caratterizzati at- traverso metodi fenotipici e genotipici per evidenziarne la biodiversità e il potenziale metabolico. In particolare, i ceppi sono stati analizzati in relazione ad alcune caratteristiche di interesse tecnologico: lipolisi, proteolisi e capacità di formare biofilm.

\section{MATERIALI E METODI}

Analisi microbiologiche dei campioni di siero-innesto Sono stati raccolti 34 campioni di siero-innesto, 21 dei quali provenivano dalla lavorazione a Trentingrana DOP di 13 caseifici dislocati in Trentino-Alto Adige (3 prelievi in tempi successivi per 4 aziende, 1 prelievo per le restanti 9 aziende) e 12 dalla lavorazione a Provolone Valpadana DOP di un unico produttore. I campioni derivavano dalla fermentazione spontanea di una parte del siero di latte proveniente dalla caseificazione del giorno precedente. Dopo la raccolta, i campioni sono stati raffreddati rapidamente $4^{\circ} \mathrm{C}$ e analizzati entro 8 ore. Per contenere eventuali effetti negativi dovuti all'elevata acidità, in tutti i campioni di siero-innesto alla raccolta è stato aggiunto carbonato di calcio sterile ( $5 \%$ v/v) (Carlo Erba Reagents, Milano, Italia). A partire da ciascun campione sono state allestite diluizioni seriali in latte scremato ricostituito (10\% p/v) (Sacco System, Cadorago, Italia), poi seminate su terreno colturale Rose Bengal Chloramphenicol (RBC) agar (Biolife Italiana, Milano, Italia) con incubazione a $25^{\circ} \mathrm{C}$ per 5 giorni.

\section{Isolamento e purificazione degli isolati} Dopo il conteggio delle colonie di lieviti, dalle piastre di RBC sono state selezionate e prelevate le colonie che presentavano caratteristiche morfologiche diverse. I ceppi così isolati sono stati purificati e mantenuti in brodo colturale Yeast Malt (Scharlab S.L., Sentmenat, Spain) con incubazione a $30^{\circ} \mathrm{C}$ per 24 ore. Una volta purificati, gli isolati sono stati osservati al microscopio ottico, sottoposti al saggio dell'enzima catalasi e conservati in Litmus Milk (Biolife Italiana) a $-18^{\circ} \mathrm{C}$.

Tipizzazione molecolare e identificazione degli isolati Gli isolati sono stati tipizzati mediante RAPD-PCR con primer M13 (5). I biotipi differenti (omologia inferiore a $90 \%$ ) sono stati successivamente identificati tramite sequenziamento parziale del dominio D1/ D2 del gene 26S rDNA secondo Kurtzman e Robnett (6) e/o della regione ITS (Internal Transcribed Spacer) secondo Schoch et al. (7).

\section{Caratterizzazione tecnologica dei ceppi}

Le attività lipolitica e proteolitica sono state determinate utilizzando come substrato rispettivamente Tributyrin Agar (Oxoid Ltd., Basingstoke, England) e Skim Milk Agar (latte scremato in polvere 5\% 
$\mathrm{p} / \mathrm{v}$, agar $3 \% \mathrm{p} / \mathrm{v}$ ), entrambi con incubazione a $30^{\circ} \mathrm{C}$ per 7 giorni. II raggio $(\mathrm{mm})$ dell'alone di lisi è stato usato come unità arbitraria per la valutazione di tali attività $(8,9)$.

\section{Produzione di biofilm}

La formazione di biofilm è stata valutata utilizzando la metodica riportata da Morandi et al. (10). I ceppi in esame sono stati inoculati in brodo colturale Sabouraud (SB) (Biolife Italiana) addizionato di glucosio $(6 \% \mathrm{p} / \mathrm{v})$ ed incubati a $30^{\circ} \mathrm{C}$ per $24 \mathrm{~h}$. La coltura fresca ottenuta è stata diluita 1:9 in SB brodo + glucosio $(6 \% \mathrm{p} / \mathrm{v})$, inoculata $(200 \mu \mathrm{L})$ in una micropiastra a 96 pozzetti ed incubata a $30^{\circ} \mathrm{C}$ per $24 \mathrm{~h}$. Terminata l'incubazione, si è proceduto all'eliminazione del brodo colturale, al lavaggio dei pozzetti con PBS e, dopo incubazione a $45^{\circ} \mathrm{C}$ per $3 \mathrm{~h}$, alla colorazione con una soluzione di safranina $(0.4 \%$ p/v). Dopo 5 min dall'aggiunta della soluzione colorante, i pozzetti sono stati lavati con acqua sterile ed asciugati a temperatura ambiente per $18 \mathrm{~h}$. Duecento $\mu \mathrm{L}$ di acido acetico (33\% p/v) sono stati aggiunti per solubilizzare l'eventuale biofilm formato e successivamente si è proceduto alla misurazione della densità ottica (OD) a $450 \mathrm{~nm}$ della soluzione ottenuta mediante spettrofotometro Infinite F200 PRO (Tecan, Grödig, Austria). La capacità di produrre biofilm da parte dei lieviti è stata classificata come debole $(\mathrm{ODNC}<\mathrm{OD} \leq 2 \times \mathrm{ODNC})$, moderata $(2 \times \mathrm{ODNC}<\mathrm{OD} \leq 4 \times$ ODNC) o forte (OD > $4 \times$ ODNC), dove ODNC è la densità ottica del controllo negativo. La produzione di biofilm è stata valutata in triplo per ciascun ceppo.

\section{RISULTATI E DISCUSSIONE}

\section{Analisi microbiologiche}

Il contenuto di lieviti nei 34 campioni di siero-innesto analizzati è mostrato in Figura 1. Risulta evidente l'elevata variabilità dei campioni prelevati da lavorazioni a Trentingrana, il cui tenore di lieviti era compreso tra 0.95 e $5.27 \log _{10}$ UFC/mL. L'andamento del grafico riflette la variabilità di concentrazione di lieviti tra le diverse realtà casearie coinvolte nello studio, ma anche la variabilità all'interno dello stesso caseificio nei vari prelievi eseguiti durante la durata della sperimentazione (un'azienda con contenuto variabile tra 1.00 e $3.60 \log _{10}$ UFC/mL in 3 prelievi successivi). In particolare, il sieroinnesto di un caseificio si è contraddistinto per il tenore di lieviti più elevato, confermato anche nei vari prelievi successivi (concentrazione compresa tra 4.58 e $5.27 \log _{10} \mathrm{UFC} / \mathrm{mL}$ ). Per quanto riguarda il siero-innesto proveniente dall'azienda produttrice di Provolone, il contenuto di lieviti è risultato variare tra 1.85 e $3.86 \log _{10} \mathrm{UFC} / \mathrm{mL}$, con i valori più bassi in corrispondenza dei prelievi effettuati duran-

\section{Tabella I. Identificazione degli isolati da siero-innesto}

Table I. Identification of isolates in whey cultures

\begin{tabular}{|c|c|c|c|c|}
\hline Genere & Specie & Provenienza & n. ceppi & n. biotipi \\
\hline Aureobasidium & pullulans & Provolone & 3 & 2 \\
\hline \multirow[t]{4}{*}{ Candida } & parapsilosis & Provolone & 1 & 1 \\
\hline & pararugosa & Trentingrana & 1 & 1 \\
\hline & pseudolambica & Trentingrana & 1 & 1 \\
\hline & spandovensis & Provolone & 1 & 1 \\
\hline Exophiala & dermatitidis & Trentingrana & 2 & 2 \\
\hline \multirow[t]{2}{*}{ Kluyveromyces } & lactis & Trentingrana & 1 & 1 \\
\hline & marxianus & Trentingrana & 16 & 7 \\
\hline Naganisha & albida & Trentingrana & 2 & 1 \\
\hline \multirow[t]{3}{*}{ Pichia } & kudriavzevii & Trentingrana & 9 & 6 \\
\hline & manshurica & Trentingrana & 3 & 2 \\
\hline & norvegensis & Trentingrana & 3 & 3 \\
\hline Rhodotorula & nothofagi & Trentingrana & 1 & 1 \\
\hline Saccharomyces & cerevisiae & Trentingrana & 8 & 7 \\
\hline Torulaspora & delbrueckii & Trentingrana & 1 & 1 \\
\hline Non identificato & & Provolone & 1 & 1 \\
\hline
\end{tabular}

te la stagione fredda. Tali risultati non sono facilmente valutabili per scarsità di dati bibliografici. Tuttavia, in uno studio sulla caratterizzazione del siero-innesto per produzioni casearie argentine, i lieviti rappresentavano contaminanti comuni, con concentrazione e biodiversità caratteristiche di ciascun caseificio, indipendentemente dai cambiamenti stagionali (4).

\section{Tipizzazione e identificazione degli isolati} L'analisi RAPD-PCR ha consentito di indagare la variabilità genetica tra le diverse specie riscontrate e la diversità all'interno della stessa specie. Gli isolati appartenenti alla stessa specie che presentavano una omologia superiore a $90 \%$ sono stati considerati ceppi identici o strettamente correlati dal punto di vista genetico (Morandi et al., 2006). La tecnica ha evidenziato una buona variabilità inter- e intra-specifica, in cui la maggior parte dei biotipi appartenenti alla stessa specie erano raggruppati nel medesimo cluster. Solo i 3 biotipi appartenenti a Pichia norvegensis non hanno forma- 


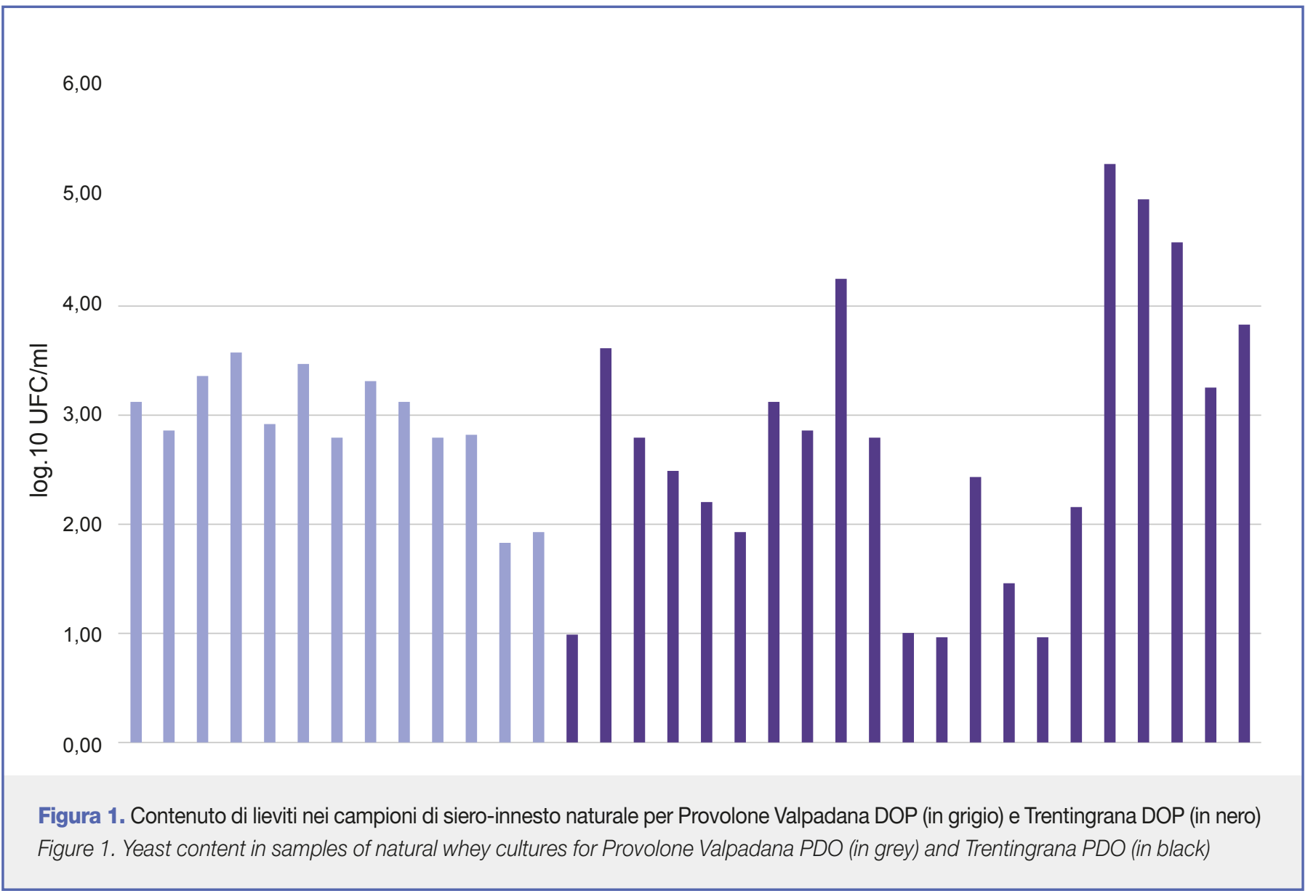

to un unico cluster e un biotipo di Saccharomyces cerevisiae è risultato geneticamente distante dagli altri biotipi della stessa specie (dati non presentati). I differenti biotipi individuati tramite RAPDPCR (omologia inferiore o uguale a 90\%) sono stati sottoposti a sequenziamento parziale del dominio D1/D2 e della regione ITS e a tutti è stato possibile attribuire una specie, ad eccezione di un ceppo isolato da siero-innesto di Provolone. I biotipi sono risultati appartenere a 9 generi e 15 specie diverse, evidenziando una complessa biodiversità microbica (Tab. I).

Le specie più rappresentate erano Kluyveromyces marxianus ( $\mathrm{n}=7$ biotipi) e Pichia kudriavzevii ( $n=6)$. È emersa una stretta correlazione tra specie e provenienza casearia, che avvalora quanto riportato da Reinheimer et al. (1995). Infatti, Aureobasidium pullulans $(n=2)$, Candida parapsilosis $(n=1)$ e C. spandovensis $(n=1)$ sono state ritrovate esclusivamente nel siero-innesto di Provolone, mentre tutte le altre specie sono risultate specifiche dei campioni da lavorazione a Trentingrana.

\section{Attività lipolitica e proteolitica}

I ceppi in esame sono stati quindi caratterizzati in funzione di alcune attività enzimatiche strettamente correlate col processo di maturazione e la qualità dei prodotti caseari (Tab II). II 90\% dei ceppi testati si è dimostrato lipolitico. Solo per 2 ceppi di $S$. cerevisiae e uno di $C$. norvegensis non è stata rilevata attività lipolitica, mentre si è evidenziata la forte attività lipolitica di $A$. pullulans e dei ceppi AY2 e TR155A appartenenti al genere Candida. Una spiccata lipolisi è stata osservata nella specie $K$. marxianus. $K$. marxianus è tra le quattro specie di lieviti comunemente impiegate come colture starter nel settore caseario, essendo in grado di influenzare la maturazione dei formaggi grazie alla propria attività proteolitica e lipolitica e alla produzione di composti aromatici volatili, tra cui etanolo e acidi carbossilici, esteri (sapore fruttato) e acetaldeide (3). Per quanto riguarda la proteolisi, il $30 \%$ dei biotipi è risultato attivo. In particolare, questa attività era evidente per i 2 ceppi di $A$. pullulans e il ceppo di C. parapsilosis da Provolone e per 2 ceppi di K. marxianus, 2 ceppi 
Tabella II. Attività lipolitica e proteolitica e produzione di biofilm dei ceppi di lieviti (-: nessuna attività; +: attività debole; ++: attività moderata; +++: attività forte)

Table II. Lipolytic and proteolytic activity and biofilm production of yeast strains (-: no activity; +: weak activity; ++: moderate activity; +++: strong activity)

\begin{tabular}{|c|c|c|c|c|c|}
\hline Genere & Specie & Ceppo & Lipolisi & Proteolisi & Biofilm \\
\hline \multirow[t]{2}{*}{ Aureobasidium } & pullulans & AY7 & ++ & ++ & + \\
\hline & & AY8 & ++ & ++ & - \\
\hline \multirow[t]{4}{*}{ Candida } & parapsilosis & AY3 & + & + & + \\
\hline & pararugosa & TR155A & ++ & - & + \\
\hline & pseudolambica & TR147D & + & - & ++ \\
\hline & spandovensis & AY2 & ++ & - & + \\
\hline \multirow[t]{2}{*}{ Exophiala } & dermatitidis & TR156A & + & - & + \\
\hline & & TR156C & + & - & + \\
\hline Kluyveromyces & lactis & TR153A & + & + & + \\
\hline \multirow[t]{5}{*}{ Kluyveromyce } & marxianus & TR110 & + & + & - \\
\hline & & TR146A & + & - & ++ \\
\hline & & TR148G & + & - & + \\
\hline & & TR159C & + & - & + \\
\hline & & TR162A & + & + & + \\
\hline \multirow[t]{8}{*}{ Pichia } & kudriavzevii & TR117 & + & - & - \\
\hline & & TR149A & + & - & + \\
\hline & & TR151A & + & - & - \\
\hline & & TR151F & + & - & + \\
\hline & & TR158A & + & - & ++ \\
\hline & norvegensis & TR143A & - & - & + \\
\hline & & TR143B & + & - & + \\
\hline & & TR143C & + & + & + \\
\hline Rhodotorula & nothofagi & TR150A & + & - & + \\
\hline \multirow[t]{5}{*}{ Saccharomyces } & cerevisiae & TR144A & + & - & + \\
\hline & & TR148A & - & + & - \\
\hline & & TR148D & - & - & - \\
\hline & & TR148F & + & + & - \\
\hline & & TR154A & + & - & + \\
\hline Torulaspora & delbrueckii & TR112 & + & - & + \\
\hline Non identificato & & AY1 & + & - & - \\
\hline
\end{tabular}

di S. cereviaisae, un ceppo di C. norvegiensis e l'unico ceppo di K. lactis da Trentingrana. I ceppi appartenenti alla specie $A$. pullulans possedevano un'attività proteolitica molto più pronunciata rispetto a quella riscontrata con gli altri ceppi considerati. Tutti i ceppi proteolitici sono risultati anche lipolitici, ad eccezione di un ceppo di S. cerevisiae. Interessante notare come queste proprietà non siano specie-, ma ceppo-specifiche. I ceppi isolati hanno, pertanto, mostrato di possedere un potenziale metabolico in grado di influenzare lo sviluppo di aromi e contribuire alla definizione delle caratteristiche sensoriali del formaggio. Queste attività enzimatiche giocano un ruolo fondamentale nella formazione di precursori dell'aroma come aminoacidi, acidi grassi e esteri. Tra gli eventi biochimici che avvengono durante la stagionatura, la proteolisi è il più complesso ed importante per lo sviluppo delle proprietà organolettiche e strutturali del formaggio (11). L'attività lipolitica propria di alcune specie di lieviti costituisce una delle proprietà necessarie per conferire le caratteristiche di tipicità ad alcune varietà di formaggi (Atanassova et al., 2016). Tuttavia, la capacità di idrolizzare i trigliceridi non ha sempre un'influenza positiva sui prodotti caseari, poiché può portare alla formazione di aromi e odori sgradevoli. Essa è, dunque, accettabile solo nel caso di formaggi a lunga stagionatura, in cui può contribuire a conferire al prodotto le sue note tipiche (13).

\section{Produzione di biofilm}

I ceppi sono stati classificati infine in base alla loro capacità di produrre biofilm (Tab II). La maggior parte di essi si è dimostrata in grado di formare biofilm, ma nessuno in maniera elevata. I ceppi con la maggior capacità di aderire al substrato sono stati TR158A (P. kudriazevii), TR146A (K. marxianus) e TR147D (C. pseudolambica). Solo otto ceppi $(27 \%)$ non sono risultati in grado di produrre biofilm, nello specifico un ceppo di $A$. pullulans, due di $P$. kudriazevii, uno di K. marxianus e tre ceppi di S. cerevisiae. Insieme a batteri lattici e batteri Gram positivi e catalasi positivi, i lieviti costituiscono i biofilm presenti su tini di legno impiegati nel processo di caseificazione di produzioni DOP tradizionali, dove il biofilm funge da "starter factory", ossia un modo efficace per inoculare in situ il latte senza utilizzare colture commerciali, avente parallelamente attività nella prevenzione dello sviluppo di agenti patogeni. $K$. marxianus, $K$. lactis e $C$. pararugosa sono tra i lieviti comunemente riscontrati in biofilm isolati da attrezzature casearie (14). 


\section{CONCLUSIONI}

Questo studio ha evidenziato nel siero-innesto di produzioni casearie italiane DOP la presenza di una popolazione di lieviti caratterizzata da un'ampia biodiversità a livello di genere e di specie. Questa biodiversità microbica, unitamente al prezioso corredo enzimatico che i ceppi oggetto di studio sono risultati possedere, concorrono alla definizione delle caratteristiche organolettiche dei prodotti caseari. Diventa, pertanto, interessante approfondire altri aspetti (ad esempio, patogenicità e attività antimicrobica) del potenziale di questi importanti protagonisti del settore caseario.

\section{CONFLITTO DI INTERESSE}

Tutti gli autori dichiarano di non aver alcun conflitto di interesse.

\section{BIBLIOGRAFIA}

1. Morandi S., Battelli G., Silvetti T., Goss A., Cologna N., Brasca M. How the biodiversity loss in natural whey culture is affecting ripened cheese quality? The case of Trentingrana cheese. LWT 2019; 115:108480.

2. Hernández A., Pérez-Nevado F., Ruiz-Moyano S., Serradilla M.J., Villalobos M.C., Martín A., Córdoba M.G. Spoilage yeasts: What are the sources of contamination of foods and beverages? Int J Food Microbiol 2018; 286:98-110.

3. Fröhlich $\nabla W y d e r$ M.T., Arias『Roth E., Jakob E. Cheese yeasts. Yeast 2019; 36(3):129-141.

4. Reinheimer J.A., Suarez V.B., Bailo N.B., Zalazar C. A. Microbiological and technological characteristics of natural whey cultures for Argentinian hard-cheese production. J Food Prot (1995); 58(7):796-799.

5. Silvetti T., Capra E., Morandi S., Cremonesi P., Decimo M., Gavazzi F et al. Microbial population profile during ripening of Protected Designation of Origin (PDO) Silter cheese, produced with and without autochthonous starter culture. LWT 2017; 84:821-831.

6. Kurtzman C.P., Robnett C.J. Identification and phylogeny of ascomycetous yeasts from analysis of nuclear large subunit (26S) ribosomal DNA partial sequences. Antonie van Leeuwenhoek 1998; 73(4):331-371.

7. Schoch C.L., Seifertb K.A., Huhndorfc S., Robertd V., Spougea J.L., Levesqueb C.A. et al. Nuclear ribosomal internal transcribed spacer (ITS) region as a universal DNA barcode marker for Fungi. PNAS 2012; 109:6241-6246.
8. Gardini F., Tofalo R., Belletti N., lucci L., Suzzi G., Torriani et al. Characterization of yeasts involved in the ripening of Pecorino Crotonese cheese. Food Microbiol 2006; 23(7):641-648.

9. Morandi S., Brasca M., Andrighetto M., Lombardi A., Lodi R. Technological and molecular characterisation of enterococci isolated from north - west Italian dairy products. Int Dairy J 2006; 16:867-875.

10. Morandi S., Cremonesi P., Capra E., Silvetti T., Decimo M., Bianchini V. et al. Molecular typing and differences in biofilm formation and antibiotic susceptibilities among Prototheca strains isolated in Italy and Brazil. J Dairy Sci 2016; 99:6436-6445.

11. Ozturkoglu Budak S. Dynamics of complex microbiota and enzymes in Divle Cave cheese and their biochemical consequences (Doctoral dissertation, Utrecht University), 2016.

12. Atanassova M.R., Fernández-Otero C., Rodríguez-Alonso P., Fernández-No I.C., Garabal J.I., Centeno J.A. Characterization of yeasts isolated from artisanal short-ripened cows' cheeses produced in Galicia (NW Spain). Food Microbiol 2016; 53:172-181.

13. Jakobsen M., Narvhus J. Yeasts and their possible beneficial and negative effects on the quality of dairy products. Int Dairy J 1996; 6:755-768.

14. Didienne R., Defargues C., Callon C., Meylheuc T., Hulin S., Montel M.C. Characteristics of microbial biofilm on wooden vats ('gerles') in PDO Salers cheese. Int J Food Microbiol 2012; 156(2):91-101. 